\title{
Paraplegia
}

\section{Compressive Mononeuropathies of the Upper Extremity in Chronic Paraplegia}

\author{
G. Davidoff, MD, R. Werner, MD, W. Waring, MD \\ Department of Physical Medicine and Rehabilitation, University of Michigan Medical \\ Center, and Rehabilitation Medicine Service, Veterans Affairs Medical Center, Ann \\ Arbor, Michigan, USA.
}

\section{Summary}

Controversy exists with regard to the actual prevalence of compressive mononeuropathies at the wrist which may occur following chronic paraplegia. Thirty one chronic paraplegics, with a mean age of 37.9 years (range 20-68 years), and mean time since injury of $9 \cdot 7$ years (range 1-28 years), were studied with a comprehensive neurologic and electrodiagnostic (EDX) assessment. No patient had any clinical or EDX evidence of a peripheral polyneuropathy. The diagnosis of a median mononeuropathy at the wrist was determined by the following criteria: (a) prolonged median sensory distal latency $>$ ipsilateral ulnar sensory distal latency $\geq 0.5 \mathrm{msec}$; (b) a median mid-palmar sensory latency $>$ ipsilateral ulnar mid-palmar sensory latency of $\geq 0.3$ msec; or (c) a median motor distal latency $\geq 1 \cdot 7$ milliseconds as compared to the ipsilateral ulnar motor distal latency. Ulnar mononeuropathy at the wrist or across the elbow was also characterised. The EDX criteria for a median mononeuropathy at the wrist was met in 55\% of subjects (24\% of these with bilateral presentations). The location of ulnar mononeuropathies included: two at the superficial sensory branch at the wrist, one at the deep motor branch at the wrist, and three patients with a conduction block across the elbow. Overall, 67\% of all patients tested had evidence of at least one mononeuropathy of the upper extremity.

There was no association between prevalence of compressive mononeuropathies and age of the patient or time since onset of injury.

Key words: Cumulative trauma disorder; Paraplegia; Compressive nerve entrapment; Upper extremity.

Previous investigators have attempted to study the prevalence and risk factors for compressive mononeuropathies of the distal upper extremity in chronic paraplegics. These studies have estimated the prevalence of carpal tunnel syndrome and/or

Correspondence to: G. Davidoff, MD, Rehabilitation Medical Service, (506/117), Veterans Affairs Medical Service, 2215 Fuller Road, Ann Arbor, Michigan 48105, USA. 
ulnar mononeuropathies at 20 to $60 \%$ (Aljure et al., 1985; Gellman et al., 1988a; Tun and Upton 1987; Gellman et al., 1988b). Limitations in the designs of these studies include substantial differences in the electrodiagnostic (EDX) protocols used for establishing the diagnosis of median or ulnar mononeuropathies at the wrist or elbow. In addition, many studies have ignored or were poorly screened for confounding factors which may influence the results of the EDX studies, including the presence of a peripheral polyneuropathy and stability of skin surface temperature.

The purpose of this study was to examine the prevalence and risk factors for compressive mononeuropathies of the upper extremity in a group of chronic paraplegics, in order to test the following hypotheses:

1. There will be a high prevalence rate of compressive mononeuropathies in the upper extremities of this patient group.

2. There will be a positive and statistically significant correlation between the duration of disability and the prevalence rate for compressive mononeuropathies of the wrist. Further, there will be a direct correlation between distal sensory and motor latency studies and the duration of disability.

3. Patients who use both canes and wheelchairs for mobility, or have a higher intensity of usage will be at greater risk for the development of compressive mononeuropathies of the upper extremities.

\section{Methods}

\section{Subjects}

Subjects were recruited from the outpatient clinics at the Veterans Affairs Medical Center, Ann Arbor, Michigan and the University of Michigan Model Systems Spinal Cord Injury Care System, Ann Arbor, Michigan during calendar years 1988 and 1989. All patients were 18 years of age or older at the time of informed consent. All patients were completely motor and sensory intact through the level of D2, and had a Frankel level of A, B or C. Informed consent was obtained according with the provisions of the Institutional Review Boards at the two study sites. Exclusionary criteria included:

1. Unequivocal evidence of a peripheral polyneuropathy, as evidenced by patient history of electrodiagnostic studies.

2. A proximal evoked median compound muscle action potential (CMAP) amplitude greater than distal CMAP indicative of a Martin-Gruber anastomosis (Thomas et al., 1967).

\section{Procedures}

After meeting the entrance criteria of the protocol and informed written consent, the subjects completed a questionnaire to assess whether the subject had symptoms of upper extremity mononeuropathy including: loss of sensation or tingling of the index and third fingers; hand or upper extremity pain which is worse during sleep and/or improves with shaking out the limb; symptoms in both hands; and hand weakness. The questionnaire also assessed avocational activities, extent of wheelchair and upper extremity use. A brief neurologic examination was 
performed including: two-point discrimination of the periungual area of the index and fifth digit; quantitative vibratory sensation of the index and fifth digit; Semmes-Weinstein monofilament testing of the periungual areas of the index and fifth digit; presence of Tinel's sign; decrease in two point discrimination following Phalen's test; and the presence of thenar atrophy and/or weakness.

The clinical diagnosis of carpal tunnel syndrome (CTS) was made if the subject had one of the following constellation of symptoms:

1. Pain or numbness in the distribution of the median nerve which worsened at night; or

2. Pain and/or numbness in the hand which did not worsen at night, in combination with either: (a) positive Tinel's sign; or (b) evidence of thenar weakness/atrophy.

Nerve conduction studies were performed using standard techniques of supramaximal percutaneous stimulation and surface recording of the following nerves bilaterally (Thomas et al., 1967):

Median sensory distal latency (stimulated at $140 \mathrm{~mm}$ and recorded at the negative peak) and sensory nerve action potential (SNAP), recorded at the index finger

- Ulnar sensory distal latency (stimulated at $140 \mathrm{~mm}$ and recorded at the negative peak) and SNAP recorded at the fifth digit.

- Median and ulnar mid-palmar (stimulated at $80 \mathrm{~mm}$ ) sensory distal latency and SNAP recorded orthodromically at the wrist

- Median motor conduction studies with stimulation at the wrist and forearm, recording at the thenar eminence. Distal latency (distal segment $=70 \mathrm{~mm}$ ), forearm conduction velocity and distal and proximal compound muscle action potentials (CMAP) were recorded

- Ulnar motor conduction studies with stimulation at the wrist, below elbow (BE) and above elbow (AE) segments, recording at the hypothenar eminence. Distal latency (distal segment $=70 \mathrm{~mm}$ ), $\mathrm{BE}$ and $\mathrm{AE}$ conduction velocities and distal and proximal CMAP were recorded

- Ulnar motor distal latency and CMAP with stimulation at the wrist, recording at the first dorsal interossei (Olney and Wilbourn, 1985)

Skin temperature was maintained $\geq 32$ degrees $\mathrm{C}$ using hydrocollator packs.

The diagnosis of a median mononeuropathy of the wrist was made if the median sensory distal latency was greater than $3.7 \mathrm{~ms}$ and met one of the other following criteria (Redmond and Rivner, 1988; Stevens, 1987):

1. The median sensory distal latency was prolonged compared to the ipsilateral ulnar sensory distal latency by greater than or equal to $0.5 \mathrm{~ms}$;

2. Median motor distal latency which was greater than ipsilateral ulnar motor distal latency by at least $1.8 \mathrm{~ms}$;

3. Median sensory distal latency on mid-palmar stimulation which was greater than ipsilateral ulnar sensory distal latency by $0.3 \mathrm{~ms}$ or greater.

The diagnostic criteria for ulnar mononeuropathy of the wrist was an ulnar sensory distal latency $>3.5 \mathrm{~ms}$, with a normal median sensory response and no evidence of an ulnar mononeuropathy at the elbow (see below); or a $>2 \mathrm{~ms}$ difference between the distal latencies of the ulnar motor nerve, recording hypothenar versus at the first dorsal interosseous muscle (Olney et al., 1983; Olney and Hanson, 1988; Shea and McClain, 1969; Olney and Wilbourn, 1985). The 
diagnostic criteria for an ulnar mononeuropathy at the elbow consisted of a $>20 \%$ drop in conduction velocity at the $\mathrm{AE}$ compared to the $\mathrm{BE}$ segments.

All patients underwent needle electromyography using a standard concentric electrode of the following muscle groups bilaterally: first dorsal interosseous, abductor digiti quinti, abductor pollicis brevis, and flexor carpi radialis to determine if axonal loss was present and to help rule out a cervical radiculopathy or other mononeuropathy which might confound the results.

All studies were performed using a TECA TD20 or Nicolet Viking.

\section{Data analysis}

Descriptive statistics were generated for all categorical and continuous measures. Prevalence rates for mononeuropathies of the median and ulnar nerves at the wrist and the ulnar nerve at the elbow were determined based on whether or not the EDX studies met the above diagnostic criteria. Simple linear regression was conducted using each of the distal sensory and motor latencies as dependent variables and patient age and time from onset of injury as independent variables. Scatter plots and residual analysis were conducted in those cases where the squared correlation coefficient was significant at an alpha $\leq 0.01$. An alpha $=0.01$ was chosen to reduce the chances for a Type I error because of the number of comparisons which were made. Two by two and three by two contingency tables were developed to compare the rates of agreement between the electrodiagnostic criteria for compressive mononeuropathy and patient complaints of pain, numbness and other symptoms consistent with the clinical diagnosis of median or ulnar compressive mononeuropathies of the upper extremities, and the clinical paradigm for CTS.

\section{Results}

Thirty two patients were screened and 31 completed the study protocol ( 1 patient was excluded because of a severe peripheral polyneuropathy). Eighty seven per cent were males, the mean age was 38 years (range 20-68 years). The mean time from onset of injury to testing was 10 years (range 1-28 years). All patients were wheelchair users. Only 4 patients (13\%) ambulated with crutches and short or long leg braces. Ninety four per cent of the subjects estimated travel greater than one kilometer per day with their wheelchair. Twenty-seven per cent engaged in wheelchair sports $(n=7)$; the mean time engaged per week in sports for these patients was 9 hours (range 2-28 hours).

Regarding clinical symptoms, 23 patients (74\%) complained of pain, numbness or paraesthesias in at least one hand; 7 patients $(23 \%)$ complained of pain or paraesthesias which worsened at night, 7 patients $(23 \%)$ had evidence of a positive Tinel's sign at the carpal canal, and 5 patients (16\%) had evidence of frank atrophy or weakness of the thenar eminence.

Based on the EDX criteria for this study protocol, 17 patients (55\%) demonstrated a compressive mononeuropathy of the median nerve at the wrist. In 4 cases this was bilateral (24\%). Two patients demonstrated a compressive mononeuropathy affecting the superficial sensory branch of the ulnar nerve at the wrist and 1 patient had involvement of the deep motor branch of ulnar nerve at the 
wrist. Three patients had a compressive neuropathy of the ulnar nerve at the elbow. Overall, 21 patients had evidence of at least one compressive mononeuropathy according to the electrodiagnostic criteria (67\%).

There was no relationship between the time since injury and the prevalence of compressive mononeuropathy (Fig. 1). Likewise there was no association between the presence of compressive mononeuropathies and the distance travelled per day in a wheelchair (Fig. 2). There was no difference in the rate of abnormal

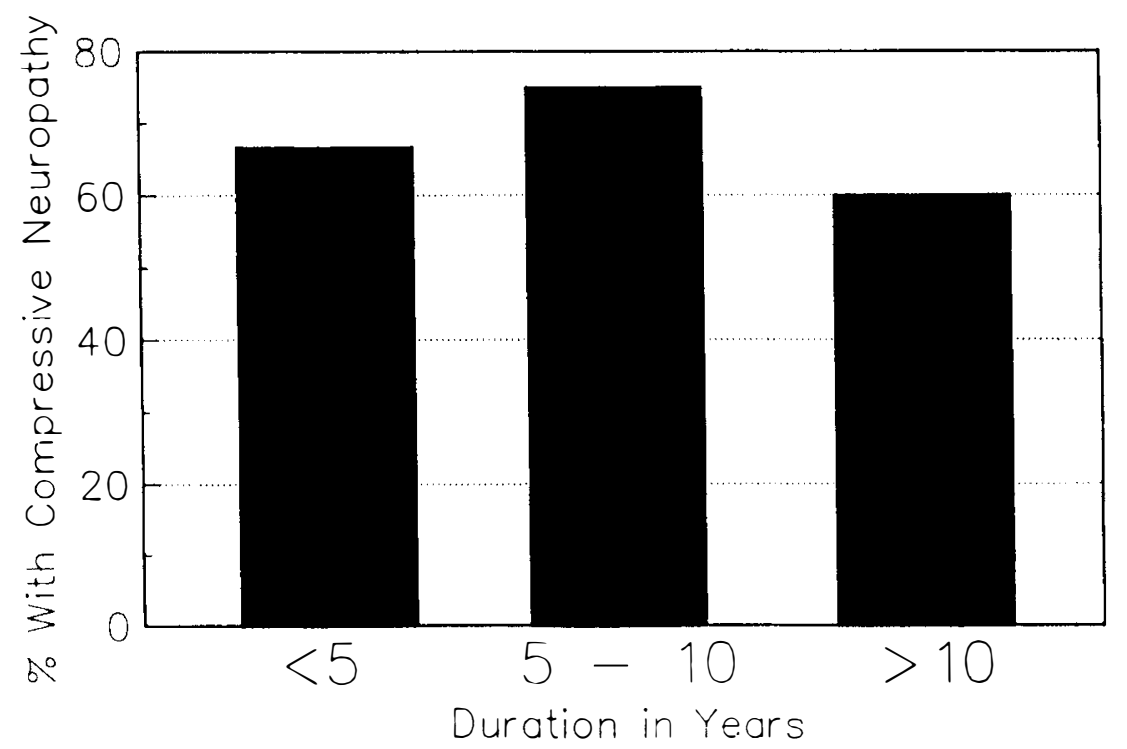

Figure 1 Duration of disability and prevalence of compressive mononeuropathy.

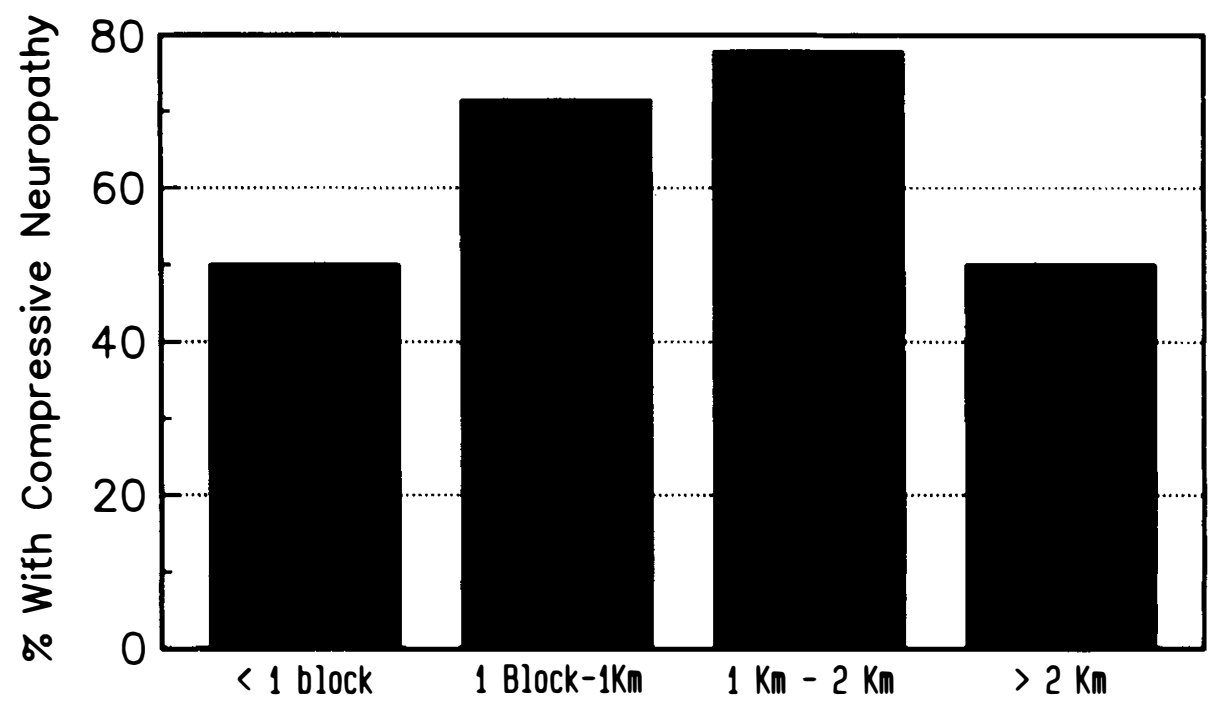

Figure 2 Wheelchair activity level and prevalence of compressive mononeuropathy. 
Table Relationship of clinical diagnosis of carpal tunnel syndrome (CTS) and electrodiagnostic (EDX) findings of median mononeuropathy at the wrist

\begin{tabular}{lcr}
\hline & EDX Findings \\
& CTS + CTS - \\
Clinical findings & CTS +15 & 10 \\
Fisher's exact test $=0.23$ (non-significant) & 4 \\
\hline
\end{tabular}

electrodiagnostic studies among those patients with or without complaints suggestive of the CTS (Table).

Finally, simple linear regression using age and time since injury as independent variables and distal latencies, conduction velocities and evoked sensory and motor amplitudes as dependent variables indicated no statistical significant relationship, with the exception of the right median motor conduction of velocity $\left(R^{2}=0 \cdot 29\right.$, $\mathrm{p}<0.005)$. However, examination of the scatter plot and residual analysis indicated no actual relationship, linear or otherwise.

\section{Discussion}

Previous authors (Blankstein et al., 1985; Kellner et al., 1986; Gellman et al., 1988b) have described the pain and disability associated with trauma to the soft tissue and peripheral nerves of upper extremities in chronic wheelchair users. It was theorised that extremes of wrist extension and wrist flexion enhance the process of degenerative joint changes as well as increase the intracarpal canal pressure, which may be associated with the development of a median mononeuropathy at the wrist (Lundborg et al., 1982; Gellman et al., 1988a; Werner et al., 1983). In the case of patients who rely on wheelchair ambulation as their main source of home and community transportation, the development of distal upper extremity mechanical and neurologic difficulties would be expected to further compromise their ability to function independently.

There has been recent interest in the development of median mononeuropathy at the wrist in paraplegic wheelchair users and other chronically disabled populations. Gellman et al., (1988a), studied 77 patients with chronic paraplegia and demonstrated that $30 \%$ met their criteria for median mononeuropathy at the wrist. There was a clear trend toward increasing prevalence of median mononeuropathy at the wrist with increasing time since injury. Aljure et al., (1985) studied 45 chronic paraplegics. Thirty of 45 patients met the criteria for median mononeuropathy at the wrist. Twenty one of these had median mononeuropathy at the wrist and evidence of ulnar nerve disease. There was a trend towards increasing prevalence of median mononeuropathy at the wrist with increased duration of disability. Werner et al. (1989) studied 148 post-polio survivors for evidence of a median mononeuropathy and found a prevalence rate of $22 \%(n=33)$. There was no association between the presence of a median mononeuropathy at the wrist with duration of disability (less than or greater than 40 years) or age (less than or greater than 50 years) or mild versus strenuous vocational activities. Stefaniwsky et al. 
(1980) found a substantially reduced $\mathrm{BE}$ and $\mathrm{AE}$ ulnar motor conduction velocity for the paraplegics as compared to twelve control subjects $(p<0.01)$. There was no association between slowing of the nerve conduction velocities and the time since onset of injury.

Several aspects of the design of the present study are unique as compared with those reviewed above. First, patients were excluded if they had a documented history or EDX studies which were consistent with the diagnosis of peripheral polyneuropathy. Second, a diagnosis of a median mononeuropathy at the wrist was only made on the basis of comparison with the ipsilateral ulnar sensory or motor studies. Care was taken to maintain skin surface temperature at $32^{\circ} \mathrm{C}$ with the use of hydrocollator packs. The use of the comparison of the mid-palmar sensory distal latencies increased the sensitivity of diagnosis. Redman and Rivner (1988) indicated a substantial false positive rate in electrodiagnostic testing for a median mononeuropathy of the wrist, when comparisons between median and ulnar distal sensory latencies were not taken into account. Finally, the protocol for the study of the ulnar nerve allowed for determination of the anatomic location of a compressive mononeuropathy of the ulnar nerve at the wrist. Screening for a Martin-Gruber anastomosis of the median nerve excluded those patients who have an anomalous innervation of the thenar eminence, which would confound the findings of this investigation.

The prevalence rate for median mononeuropathy at the wrist in this study of 31 patients was $54 \%$. This is similar to the findings of Aljure et al. (1985) who reported a prevalence rate of $64 \%$. These results differ from those of Gellman et al. (1988a) who reported a prevalence rate of $30 \%$. These differences may be a result of the more sophisticated criteria for diagnosis based on electrodiagnostic findings. In addition, this study is the first to stratify ulnar mononeuropathies of the wrist versus elbow in this patient group. The criteria are far more rigorous than those of Stefaniwsky et al. (1980). There are two striking findings in our study: (1) the lack of relationship between EDX parameters, and time since onset of injury; and (2) the lack of correlation between patient complaints and their EDX findings (Table I). Previous studies do not agree with regard to consistent evidence for a positive relationship between these factors. Our findings may indicate that patients who are able to propel their wheelchair for more than a kilometer per day following their initial rehabilitation and are at least 1 year post injury, are at equal risks to develop a compressive mononeuropathy of the upper extremity within the first 5 to 10 years following injury at any time thereafter.

Evidence from the studies of Werner et al. (1983), Lundborg et al. (1982) and Gellman et al. (1998a) suggest that it is the chronic compression of the median nerve by increased carpal canal pressure during extremes of wrist flexion and wrist extension that lead to development of a median mononeuropathy at the wrist. Likewise, compressive mononeuropathies of the ulnar nerve at the wrist or elbow may be a function of repetitive trauma in the course of propelling a wheelchair or performing transfers. Werner et al. (1989) estimates a 4-fold increased risk for developing a median mononeuropathy at the wrist in those patients who had combined use of a wheelchair and cane/crutch. The chronic use of wheelchairs and dependence upon upper extremities for transfers and other mobility related activities may cause these subjects to develop a cumulative trauma disorder similar to that described in the industrial setting. Further studies are needed to determine 
the long term implications of EDX abnormalities in the absence of functionally limiting patient complaints of pain and/or weakness, as well as the apparent lack of relationship between EDX findings and duration of disability.

\section{Acknowledgements}

This study was supported by a Clinical Investigator Development Award to Professor Davidoff from the National Institute of Neurological Disorders and Stroke, Bethesda, Maryland (NS-01120-03); the Clinical Research Center of the University of Michigan Hospitals, Ann Arbor, Michigan (NIH grant no. 5-MO1-RR00042); the Kenny Michigan Rehabilitation Foundation, Southfield, Michigan and a Research Training Grant from the National Institute on Disability and Rehabilitation Research, Washington, DC, (H133-P80017). Kathryn Stolp-Smith, MD, MS helped design the EDX protocol. Secretarial assistance was skillfully provided by Syed Islam, MD.

\section{References}

Aljure J, Eltorai I, Bradley WE et al. 1985 Carpal tunnel syndrome in paraplegic patients. Paraplegia 23:182-186.

Blankstein A, Shmueli R, WeIngaRTEn I et al. 1985 Hand problems due to prolonged use of crutches and wheelchairs. Orthopaedic Review 14:29-34.

Gelberman RH, Hergenroeder PT, Hargens AR, et al. 1981 The carpal tunnel syndrome. Fournal of Bone and Foint Surgery 63A:680-683.

Gellman H, Chandler DR, Petrasek J et al. 1988a Carpal tunnel syndrome in paraplegic patients. Fournal of Bone and Foint Surgery 70:517-519.

GellmaN H, SIE I, WATERS RL 1988b Late complications of the weight bearing upper extremity in the paraplegic patient. Clinical Orthopedics 253:132-135.

KellNER WS, FelsENTHAL G, ANDERSON JM et al. 1986 Carpal tunnel syndrome in the nonparetic hands of hemiplegics: Stress induced by ambulatory assistive devices. Orthopaedic Review 15: 87-90.

Lundborg G, Gelberman RH, Minter-Convery M et al. 1982 Median nerve compression in the carpal tunnel-functional response to experimentally induced controlled pressure. Fournal of Hand $\operatorname{Surg}(A m)$ 7:252-259.

OLNEY RK, HANSON M 1988 AAEE case report \# 15: Ulnar neuropathy at or distal to the wrist. Muscle and Nerve 11:828-832.

OLNEY RK, WILBOURN AJ 1985 Ulnar nerve conduction study of the first dorsal interosseous muscle. Archives of Physical Medicine and Rehabilitation 66:16-18.

OLNEY RK, WilbouRN AJ, MilleR RG 1983 Ulnar neuropathy at or distal to the wrist. Neurology 33: 185.

REDMOND MD, RIVNER MH 1988 False positive electrodiagnostic tests in carpal tunnel syndrome. Muscle and Nerve 11:511-517.

Shea JD, McClain EJ 1969 Ulnar nerve compression syndromes ai and below the wrist. The fournal of Bone and foint Surgery 51-A:1091-1103.

STEFANIWSKy L, Bilowit DS, PRASAD S 1980 Reduced motor conduction velocity of the ulnar nerve in spinal cord injured patients. Paraplegia 18:21-24.

STEVENS JC 1987 AAEE. Minimonograph \#26: The electrodiagnosis of carpal tunnel syndrome. Muscle and Nerve 10:99-113.

Thomas JE, LAMBERT EH, CSEUZ KA 1967 Electrodiagnostic aspects of median mononeuropathy at the wrist of the carpal tunnel syndrome. Archives of Neurology 16:635-641.

TUN CG, UPTON 1987 The paraplegic hand: electrodiagnostic studies and clinical findings. Presentation at the Annual Meeting of the American Spinal Injury Association.

WeRner CO, ElmQuist D, Ohlin P 1983 Pressure and nerve lesions in the carpal tunnel. Acta Orthopedica Scandinavia 54:312-316.

WERNER R, WARING W, DAVIDOFF G 1989 Risk factors for median mononeuropathy of the wrist in postpoliomyelitis patients. Archives of Physical Medicine and Rehabilitation 70:464-467. 